\title{
Erratum to: Design and implementation of a 700-2,600 MHz RF SiP module for micro base station
}

\author{
Yi He $\cdot$ Fengman Liu $\cdot$ Fengze Hou $\cdot$ Peng Wu . \\ Jun Li $\cdot$ Liqiang Cao $\cdot$ Dongkai Shangguan
}

Published online: 15 October 2014

(C) Springer-Verlag Berlin Heidelberg 2014

\section{Erratum to: Microsyst Technol}

DOI 10.1007/s00542-014-2314-5

The acknowledgement section was wrongly published in the original publication. The correct version is given below.

Acknowledgments The authors wish to acknowledge the support from CAS/SAFEA International Partnership Program for Creative Research Teams, Hundred Talent Program of the CAS and the National Natural Science Foundation of China Project No. 61474140.

The online version of the original article can be found under doi:10.1007/s00542-014-2314-5.

Y. He $(\bowtie) \cdot$ F. Liu $\cdot$ F. Hou $\cdot$ P. Wu $\cdot$ J. Li $~ \cdot$ L. Cao $\cdot$ D. Shangguan Institute of Microelectronics of Chinese Academy of Sciences, No. 3, Beitucheng West Road, Chaoyang, Beijing 100029, China e-mail: heyi@ime.ac.cn

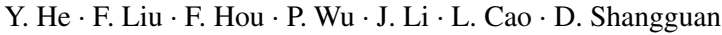
National Center for Advanced Packaging (NCAP China), Building D1, 200 Linghu Boulevard, Wuxi 214135, Jiangsu, China 